Conf- $711106--64$

PATL-SA- -19643

DE92 006471

\title{
AUTOMATED PHANTOM ASSAY SYSTEM
}

D. R. Sisk

L. L. Nichols

P. C. Olsen

November 1991

Presented at the 1991 IEEE Nuclear Science Sympos ium

November 5-8, 1991

Santa Fe, New Mexico

Work supported by

the U.S. Department of Energy

under Contract DE-ACO6-76RLO 1830

Pacific Northwest Laboratory

Richland, Washington 99352

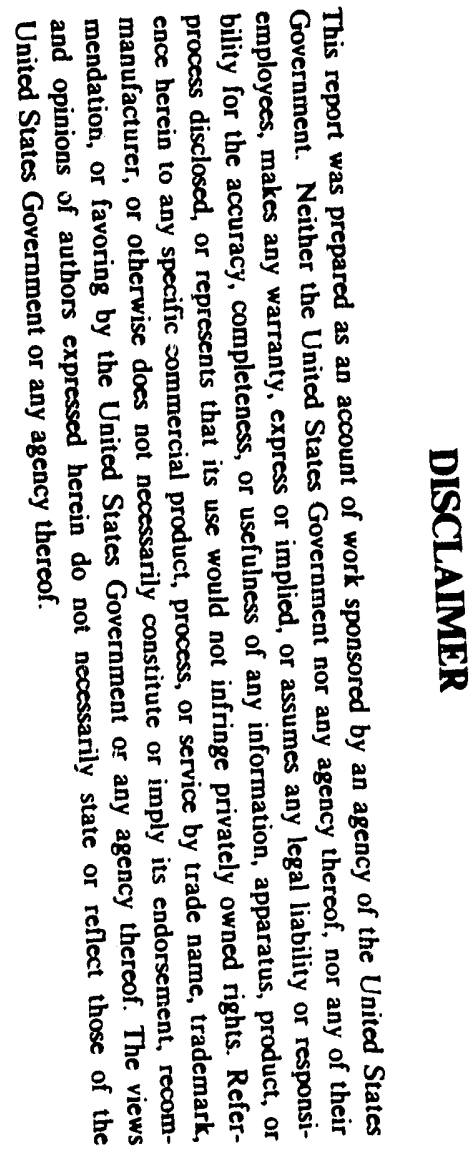

MASTER 


\title{
AUTOMATED PHANTOM ASSAY SYSTEM
}

\author{
D.R. Sisk, L.L. Nichols, P.C. Olsen \\ Pacific Northw'est Laboratory ${ }^{1}$ \\ Richland, WA 99352
}

\begin{abstract}
Absiract
This paper describes an automated phantom assay system dsieloped for assaying phantoms spiked with minute qu:ntities of radionuclides. The system includes a computercontrolled linear-translation table that positions the phantom at exact distances from a spectrometer. A multichannel analyzer (MCA) interfaces with a computer to collect gamma spectral data. Signals transmitted between the controller and MCA synchronize data collection and phantom positioning. Measured data are then stored on disk for subsequent analysis. The automated system allows continuous unattended operation and ensures reproducible results.
\end{abstract}

\section{INTRODUCTION}

Calibration and accreditation of in vivo assay equipment at Department of Energy (DOE) facilities require the measurement of human organ phantoms spiked with minute quantities of transuranics and fission products. To make the phantoms, a precise quantity of radionuclides is added to an epoxy mixture. Then the mixture is poured into a mold and allowed to harden.

The quantities of radionuclides used in the production of phantoms are certified according to National Institute of Standards and Technology (NIST) standards. To maintain the integrity of the calibration, the amount of radioactive material in completed phantoms must be verified following production.

One method of verification requires the measurement of the excess material generated during the manufacture of the phantom. The quantity of radionuclides in the excess material is subtracted from the original quantity to deter-

\footnotetext{
1 Operated by Battelle Memorial Institute for the U.S. Department of Energy under Contract DE-ACO6-76RLO 1830.

2 The identification of equipment used for this study is intended neither as an endorsement nor as a recommendation for its use.
}

mine the amount of radioactivity contained in the phantom. However, because of the small quantities of radioactivity involved in the manufacturing of the phantoms, satisfactory precision is difficult to obtain with this method [1]. Another method involves direct measurement of the phantom by destructive analysis. Destructive analysis is necessary because the large size: of the phantoms precludes the use of assays based on poini-source techniques.

A technique that overcomes the difficulties of both of the aforementioned methods has developed from work by Evans [2]. This technique, which requires eight measurements with a high-grade gamma spectrometer at various distances, uses inverse $r$-squared relationships to extrapolate phantom assays to an equivalent point-source geometry. In addition, the technique attempts to account for the attenuation of gamma rays in the phantom. However, even with a highly efficient detector, the time required for each measurement can exceed 24 hours because of the small concentrations of radionuclides.

This paper describes a computerized system developed by the Pacific Northwest Laboratory (PNL) $)^{(a)}$ for automating phantom assay measurements. The system includes a computer-controlled linear translation table that positions the phantom at exact distances from a high-efficiency (70\%) germanium detector. The computer controls the positioning of the phantom as well as the acquisition of data. The following sections describe the design and performance of the automated phantom assay system.

\section{SYSTEM CONFIGURATION}

The automated phantom assay system consists of a highefficiency germanium gamma spectrometer, a linear translation table and controller, and a personal computer with an internal multichannel analyzer.

The spectrometer is an ORTEC model GEM-70205-P with a 30-liter liquid nitrogen dewar ${ }^{2}$. The germanium crystal used for the detector is $63.2 \mathrm{~mm}$ long and $74.4 \mathrm{~mm}$ in diameter. The detector's efficiency', determined from the manufacturer's calibration, is approximately $70 \%$. The detector is enclosed by a lead cave approximately $10 \mathrm{~cm}$ 
thick. The front face of the detector is positioned at the open end of the cave. Signal cables for the detector and a hose to fill the liquid nitrogen dewar are routed through a pass-through in the cave (Figure 1).

The linear translation table is a Daedal model 506481S modified to include limit and home switches. The table is equipped with a linear position encoder with a resolution of $0.000254 \mathrm{~cm}$. The table has a travel distance of approximately $107 \mathrm{~cm}$ and a positioning accuracy of $\pm 0.000508 \mathrm{~cm}$. The motion of the translation table is controlled by a Daedal model MC2000 controller, which provides both manual as well as programmable control by a host computer via a RS232-C communication interface. The controller can be programmed to perform a series of operations repetitively. In addition, the controller can be programmed to suspend operations until a TTL (transistor-transistor logic) signal is detected at one of the controller's trigger inputs. The controller can also be programmed to transmit a TTL signal to indicate that an operation has been completed.

An IBM personal computer provides for system control and data storage while a Cauberra model $\mathbf{4 6 1 0}$ multichannel analyzer (MCA) initiates and controls the collection of gamma spectral data. The MCA is a computer card that plugs into an expansion slot of the personal computer. Pulses from the spectrometer's preamplifier are routed to an external spectroscopy amplifier and then to an analog-todigital converter $(A D C)$. Data from the $A D C$ is then transmitted to the MCA. Computer software supplied with the MCA is used to display and analyze the gamma spectrum. The computer software provides a utility for programming a sequence of MCA operations and allows the user to select the number of iterations of the sequence. The MCA can also be programmed to transmit and receive TTL-compatible signals over external signal lines.

To perform the phantom assays, the translation table controller and MCA were interfaced via two signal lines. One of the signal lines was used by the MCA to transmit a signal to the trigger input of the controller. The other signal line was used by the controller to indicate to the MCA that a move was complete. Consequently, a rquisition of gamma spectra could be synchronized with the movernent of the translar:on table. Figure 2 shows a block diagram of the system.

A program on the personal computer configured the translation table controller prior to each measuremunt process. The computer initialized the speed, acceleration, and state of the trigger and output signal lines. After moving the table to a predefined origin, the computer positioned the table at the first measurement point. The computer then programmed the controller to perform a sequence of operations. The number of iterations of the sequence was established by the user. In the sequence, the controller would remain in a wait state until a signal was received on the trigger input. This signal was generated by the MCA when a spectrum had been collected. Once the trigger signal had been received, the controller would position the table at the next measurement point. The controller would then transmit a signal to the MCA to indicate that the move was complete.

The computer software accompanying the MCA controlled the remainder of the measurement process. The software was programmed to perform a series of tasks, which were repeated for each measurement point. These tasks inclucied collecting the gamma spectrum, saving the spectrum to floppy disk, and signalling the controller to position the phantom at the next measurement point. The MCA then suspended operations until a confirmation signal was received from the controller. The confirmation signal indicated the completion of the movement of the phantom to the next measurement position. The cycie was then repeated for each measurement point.

\section{SYSTEM PERFORMANCE}

In order to provide satisfactory error statistics, the gamma spectrum at a particular measurement point was measured until the integrated count under that particular gamma energy peak was at least 10,000 counts. Therefore, the counting time per measurement point was proportional to the amount of radioactivity in the phantom. A full series of measurements (eight measurement points) with a phantom containing radioactivity on the order of several nanocuries took approximately one week. Results from a sequence of measurements of a lung phantom spiked with ${ }^{137} \mathrm{Cs}$ are shown in Figure 3. Note that the product of the count rate and the square of the distance is approximately constant with the distance. This situation is necessary if the amount of radioactivity in the phantom is to be accurately estimated. Similar measurements with a point source of known activity resulted in an error on the order of one tenth of a percent.

\section{CONCLUSIONS}

The automated phantom assay system permits unattended operation and ensures reproducible results. In conjunction with the inverse $r$-squared technique, the system helps to obtain precise results in phantom assays. The system will be used to assay lung phantoms contained in the PNL Phantom Library. In addition, the system will be used to verify organ burdens for the upcoming DOELAP In Vivo Bioassay Accreditation pilot test program, which the DOE Radiological and Environmental Sciences Laboratory at Idaho Falls, Idaho, is undertaking. In the future, the system will be used in measuring and verifying the uniformity of distribution of radioactivity in phantoms. This is necessary if phantoms are to accurately simulate actual human organs. 


\section{REFERENCES}

[1] Olsen, P.C, L.L Nichols, D.R Sisk, and R.M. Loesch. 1991. The Pacific Northwest Laboratory In Vivo Phantom Library Calibration Program." Presented at the 37th Annual Conference on Bioassay, Analytical and Environmental Radiochemistry, Ottawa, Ontario. Pacific Northwest Laboratory, Richland, Washington.

[2] RD. Evans and RO. Evans, "Studies of Self-Absorption in GammaRay Sources," Reviews of .rivdern Physics, pp. 305-326, 1948.

\section{FIGURES}

Figure 1 Measurement geometry showing translation table, lung phantom, and detector face.

Figure 2 Block diagram of automated phantom assay system.

Figure 3 Plot of the Product of the Count Rate and Square of the Measurement Distance as a Function the Measurement Distance for a Lung Phantom Spiked with ${ }^{137}$ Cs. 


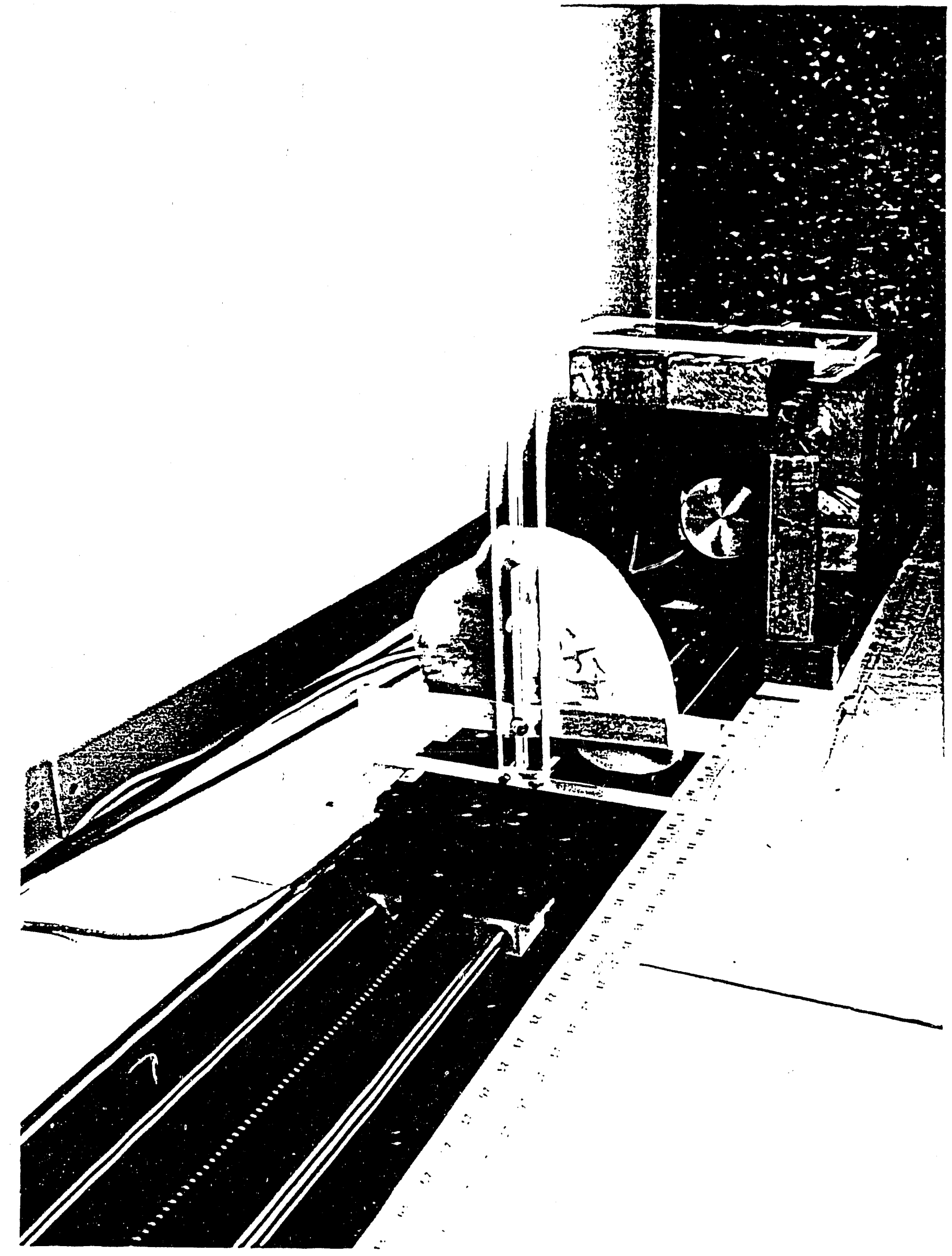

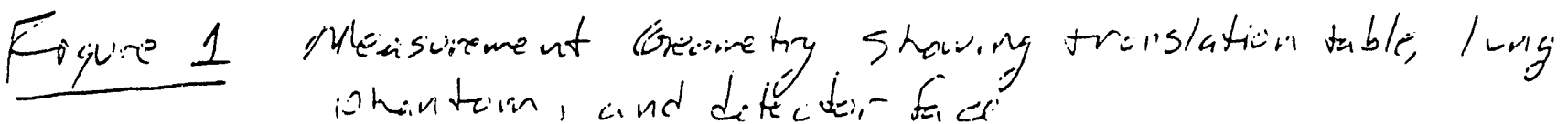




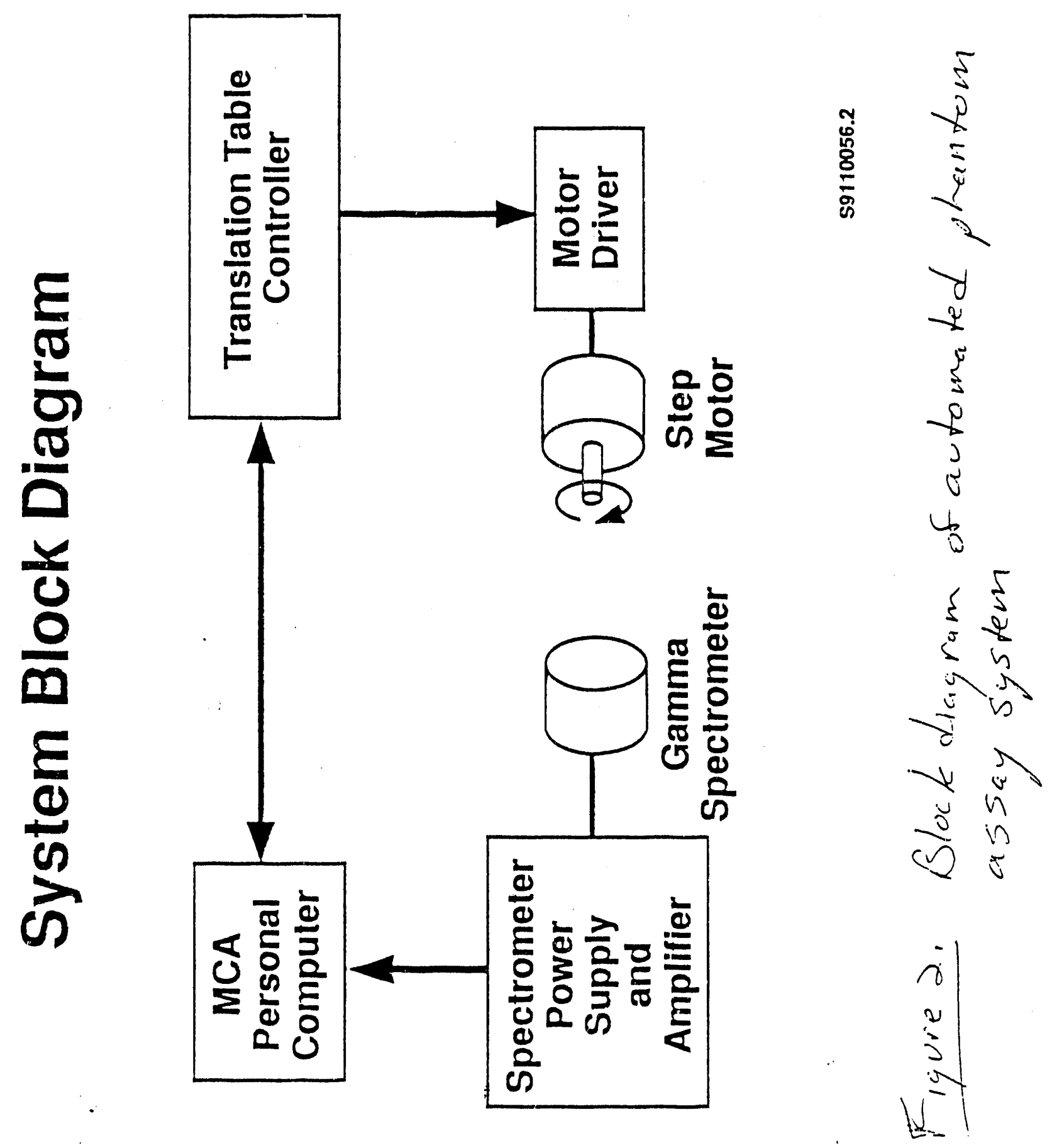


$\infty$

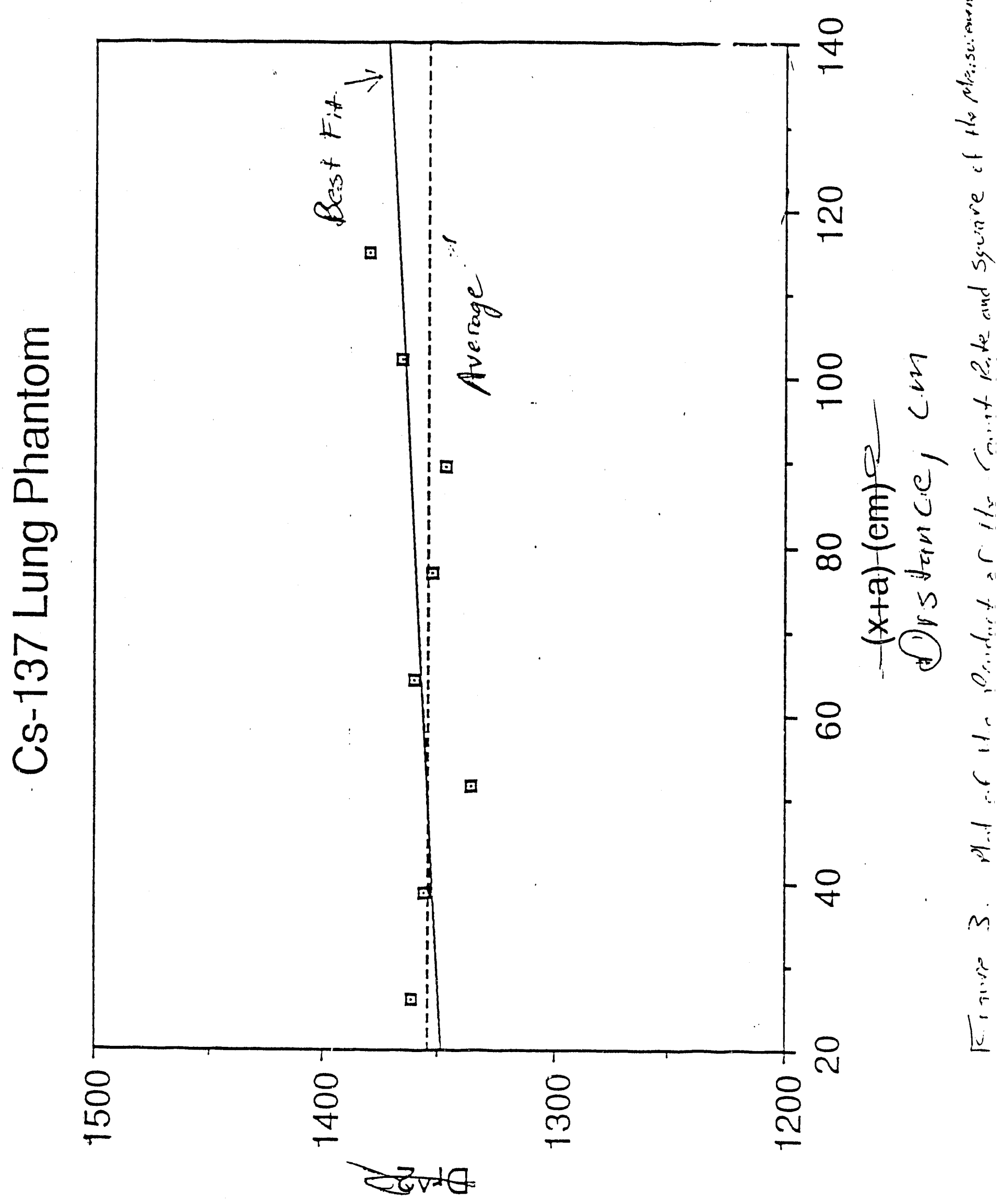



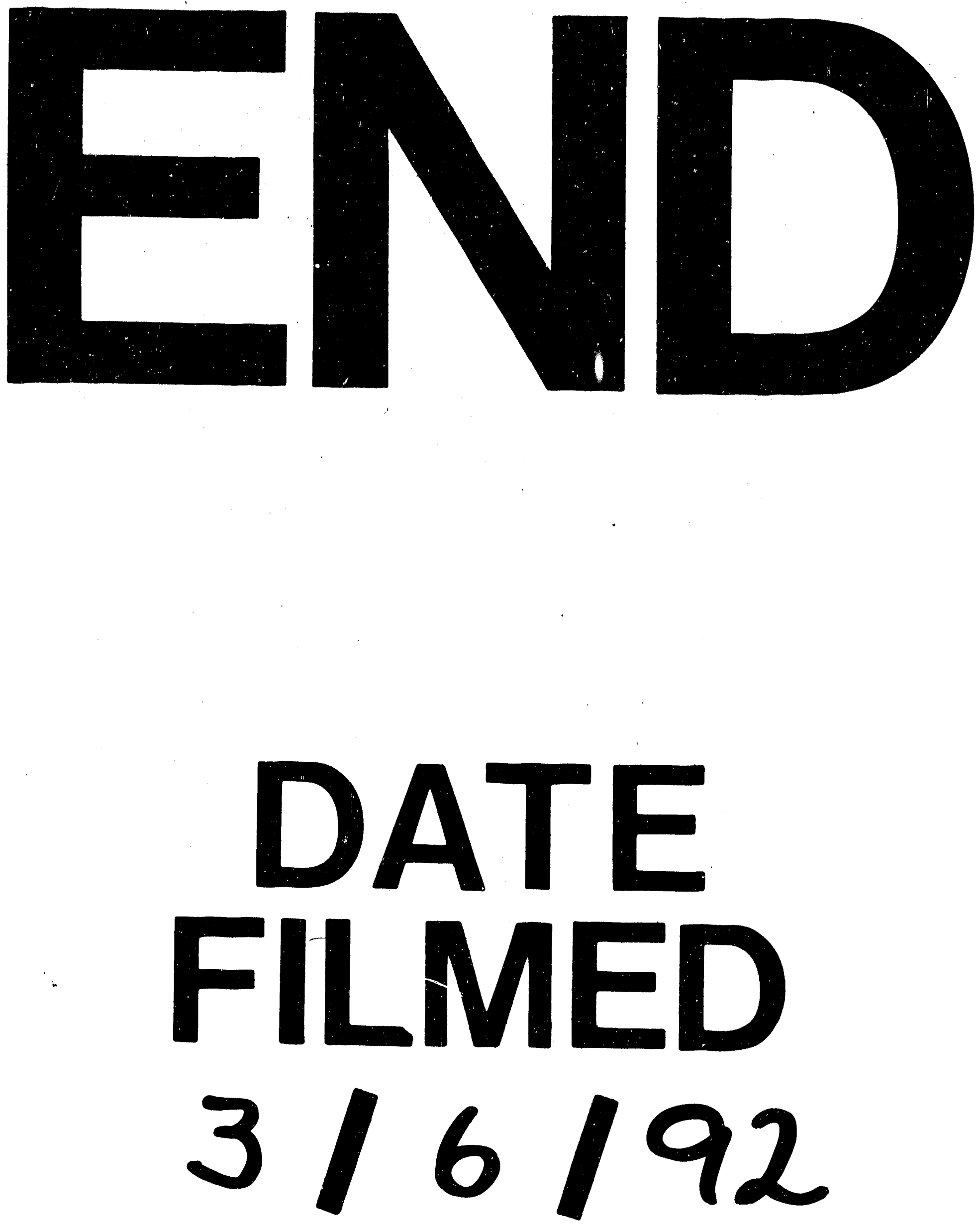
